EXTENDED REPORT

\title{
Influence of LASIK on scanning laser polarimetric measurement of the retinal nerve fibre layer with fixed angle and customised corneal polarisation compensation
}

\author{
G Holló, A Katsanos, P Kóthy, A Kerek, I Süveges
}

Br J Ophthalmol 2003;87:1241-1246

Background/aim: Retinal nerve fibre layer thickness (RNFLT), as measured with scanning laser polarimetry using the fixed angle corneal polarisation compensator (SLP-F), has been found to be reduced after uncomplicated laser assisted in situ keratomileusis (LASIK) compared to the pre-LASIK measurement. Since this virtual RNFLT thinning is attributed to the corneal changes induced by the LASIK, the authors investigated whether customised corneal polarisation compensation (SLP-C), which compensates for the actual corneal polarisation during each measurement, can avoid the LASIK induced, virtual changes of the polarimetric RNFLT values.

Methods: Scanning laser polarimetry using both the SLP-F and SLP-C methods (GDx-Access, software version 5.0) was performed on 15 consecutive healthy subjects with no eye disease who underwent LASIK for ametropia correction. The SLP measurements were performed before the surgery, then on day 1 and day 6 after LASIK. Thickness data from images of one randomly selected eye per subject were analysed using the ANOVA and Scheffe multiple comparison tests.

Results: Superior maximum, inferior maximum, normalised superior area, and normalised inferior area (SLP parameters representing the RNFLT at the superior and inferior poles of the optic nerve head) remained unchanged with SLP-C (ANOVA, $\mathrm{p}>0.05$ ) but decreased (superior maximum, normalised superior area, Scheffe test, $\mathrm{p}<0.05$ ) or tended to decrease (inferior maximum) after LASIK, when measured using SLP-F. In contrast, certain other parameters-namely, superior ratio and inferior ratio (representing the ratios between the superior or the inferior sector and the temporal sector), maximal modulation, and ellipse modulation decreased with SLP-C (Scheffe test, $p<0.05$ ), but remained stable with SLP-F (ANOVA, $\mathrm{p}>0.05$ ) after LASIK. Superior to nasal ratio, symmetry of the superior and inferior RNFLT as well as the parameter showing the probability of having glaucoma (called "the number") remained unchanged with both types of corneal compensation (ANOVA, $p>0.05$ ). With SLP-C the parameter ellipse average thickness increased after LASIK (Scheffe test, $p=0.021$ ). No parameter value altered between day 1 and day 6 after LASIK, for either method.

See end of article for authors' affiliations

Correspondence to: Gábor Holló MD, ist Department of Ophthalmology, Semmelweis University, $\mathrm{H}-1083$, Budapest, Tömö u, 25-29, Hungary; hg@szem1.sote.hu

Accepted for publication 12 February 2003

Conclusion: The results suggest that the LASIK induced decrease of the polarimetric RNFLT, which is consistently detected with polarimeters when using the fixed angle corneal polarisation compensator, is due to alterations of the corneal polarisation. The use of customised corneal polarisation compensation avoids this virtual decrease of the polarimetric RNFLT. However, our results suggest an increase of the measured retardation in the temporal quadrant of the SLP-C image after LASIK. Since ratios of parameters using the temporal RNFLT in the denominator are important in the polarimetric glaucoma diagnosis algorithm, their decrease as a consequence of using SLP-C needs further investigation.

$\mathrm{S}$ canning laser polarimetry (SLP) of the retinal nerve fibre layer has become a widely used non-invasive technique for glaucoma diagnosis ${ }^{1-5}$ and for follow up monitoring. ${ }^{6-8}$ The principle of polarimetry is the measurement of the optical retardation of the illuminating laser beam after its reflection from the retina. ${ }^{9}$ Since not only the retinal nerve fibres around the optic nerve head, but also the parallel collagen fibres of the central cornea, influence the measured retardation, and thus the software calculated retinal nerve fibre layer thickness (RNFLT) values, compensation for the corneal polarisation is of great clinical importance. ${ }^{1011}$

Interindividual differences in corneal retardation are significant, ${ }^{12}{ }^{13}$ and influence the diagnostic sensitivity of SLP with instruments using the fixed angle corneal polarisation compensator. ${ }^{12-15}$ The polarisation magnitude of the compensator is $60 \mathrm{~nm}$, and its slow axis is set to $15^{\circ}$ nasally downwards ${ }^{13}$ since many, but not all, corneas have a similar polarisation axis. ${ }^{13}$ In fact, the distribution of the slow axis of corneal polarisation is widely variable among individuals. ${ }^{12}{ }^{13}$
This means that the corneal effect cannot be neutralised in very many eyes if the fixed angle compensator is used..$^{12-16}$

In addition to its impact on the initial diagnostic accuracy of SLP, fixed angle corneal compensation is disadvantageous for longitudinal follow up evaluation in cases where the polarising properties of the cornea are modified. The polarisation axis of the normal cornea has been found to be stable over a period of at least a year, ${ }^{17}$ but corneal polarisation seems to be significantly influenced by laser assisted in situ keratomileusis (LASIK), a refractive surgery widely used to correct ametropia. ${ }^{18}$ Polarimetric RNFLT as measured with instruments using the fixed corneal compensator (SLP-F) has been consistently reported to be decreased after uncomplicated LASIK, compared to the pre-LASIK result. ${ }^{18-23}$ At the same time the RNFLT as measured with alternative methods (scanning laser tomography and optical coherence tomography) remained stable. ${ }^{21}$ This suggests that the polarimetric alterations in such cases are artefacts due to the alterations of the corneal polarisation, which 
is probably caused by the post-LASIK corneal healing process. $^{24-29}$

In order to solve the problem of compensation for corneal polarisation in SLP a new customised (individualised) corneal compensation technique (SLP-C) has recently been introduced in polarimetry. In SLP-C a polarimetric image of the macula is acquired as the first step of the measurement. ${ }^{12}{ }^{14-16}$ Since the fovea is free from retinal ganglion cell axons, the resulting polarimetric image of the macular area represents the sum of the polarimetric effects only of the cornea, the lens, and the Henle fibre layer. The second step is imaging the peripapillary retinal nerve fibre layer. Data derived from the macular image are then automatically subtracted from the peripapillary polarimetric image. The resulting "corrected" image of the peripapillary retinal nerve fibre layer is analysed automatically by the software.

In addition to the potential advantage of customised corneal compensation in the polarimetric initial diagnosis of glaucoma, SLP-C may offer the possibility of compensation for transient changes of corneal polarisation. This may be useful for longitudinal follow up evaluations when the corneal polarisation of the particular subject alters during the follow up period. In order to study the clinical value of SLP-C in this respect, we measured the polarimetric RNFLT of 15 healthy eyes before and after uncomplicated LASIK, using both the SLP-F and SLP-C corneal compensation techniques in each case.

\section{PATIENTS AND METHODS}

The clinical investigation protocol was approved by the ethics committee of the university. Fifteen consecutive healthy white subjects (mean age 37.0 (SD 15.3) years, range 19-75 years) without any ocular disease or abnormality except for ametropia were enrolled the study. The patients had no history of eye disease. Uncorrected visual acuity and best spectacle corrected visual acuity were assessed in the preoperative period. Slit lamp examination, Goldmann applanation tonometry, funduscopy, automated refractometry with and without pupil dilation and cycloplegia, corneal topography, and pachymetry (Orbscan II Z, Bausch \& Lomb, Rochester, NY, USA) were also performed before the LASIK intervention. All eyes were evaluated as being normal. The central corneal thickness was greater than $500 \mu \mathrm{m}$ in each eye. Intraocular pressure in each eye was consistently below $22 \mathrm{~mm} \mathrm{Hg}$, and the optic nerve heads showed no pathological alterations when examined using a 90 dioptre noncontact aspherical lens and a slit lamp for stereoscopic viewing. Preoperative spherical refractive error for the 12 myopic eyes varied between $-1.00 \mathrm{D}$ and $-6.75 \mathrm{D}$ (mean -4.00 (SD 1.64) D). The range for the three hyperopic eyes was between +3.25 D and +5.50 D (+4.25 (1.15) D). The preoperative cylindrical refractive error for the whole group was between $-1.0 \mathrm{D}$ and $+0.5 \mathrm{D}(-0.13(0.40) \mathrm{D})$. Patients were asked not to wear their contact lenses for at least 4 weeks before the preoperative assessment.

All the LASIK procedures were performed by the same surgeon on the same day, using an identical technique in each case with respect to the perioperative care and the flap manipulations, as summarised below. Before surgery the upper and lower eyelids were treated with povidone-iodine antiseptic preparation, and one drop of anaesthetic solution (oxybuprocaine $0.4 \%$ ) was applied to the corneal surface. The cornea was irrigated with balanced salt solution (BSS), and the appropriate diameter suction ring ( $8.5 \mathrm{~mm}$ for myopic and $9.5 \mathrm{~mm}$ for hyperopic eyes) was positioned on it to fix the eye during the flap preparation. After creating the vacuum, which was built up gradually, a Barraquer tonometer (Carriazzo-Barraquer Mario SA, Antony, France) was used to check that the intraocular pressure was in the correct range (>65 mm Hg). A corneal flap with the hinge at the 12 o'clock position was created using a Hansatome microkeratome (Bausch \& Lomb, Rochester, NY, USA). The flap thickness was $160 \mu \mathrm{m}$ or $180 \mu \mathrm{m}$, depending on the original corneal thickness and the planned stromal ablation depth. The suction ring was disengaged, the flap was gently lifted with a spatula. An ablation zone of $6 \mathrm{~mm}$ diameter was applied in all cases. The ablation was performed using a Technolas 217 flying spot excimer laser (Bausch \& Lomb, Rochester, NY, USA) using the PlanoScan software and an active eye tracker system. The beam parameters were: beam diameter $2 \mathrm{~mm}$, fluence $120 \mathrm{~mJ} / \mathrm{cm}$, repetition rate $50 \mathrm{~Hz}$. After the laser treatment the flap was repositioned and the surface and the underside were irrigated with BSS, followed by drying out. A striae test was performed to check that the flap had properly adhered. At the end of the procedure one drop each of antibiotic (ciprofloxacin) and steroid (fluorometholone) solutions were instilled. Patients were checked 30 minutes after surgery, the next day, and 1 week later. During this period the antibiotic and steroid drops were applied three times a day.

Scanning laser polarimetry of the retinal nerve fibre layer was performed by the same investigator (AK) without pupil dilation using the GDx nerve fibre analyser access instrument (Laser Diagnostic Technologies, Inc, San Diego, CA, USA) with software version 5.0. This instrument developed for glaucoma screening contains a conventional fixed angle (slow axis $15^{\circ}$ nasally downwards) corneal polarisation compensation unit for classic type measurements (SLP-F); but the software also permits customised corneal compensation for image acquisition in the "customised mode" (SLP-C). Both types of measurement can be performed during the same measurement session, immediately after one another. The non-invasive polarimetric measurement technique with fixed corneal polarisation compensation has been described in detail elsewhere. ${ }^{1-9}$ In brief, a beam of $780 \mathrm{~nm}$ polarised laser light is projected onto the retina by the instrument. The birefringent, parallel nerve fibres cause retardation in the polarised light passing through them, and this retardation of the light reflected from the retina is measured automatically by the detector unit of the instrument. The degree of the retardation corresponds to the thickness of the RNFL, and is analysed by the built-in polarisation detection unit. RNFL thickness is automatically calculated for each pixel, and is then imaged for the retinal area in a colour coded manner. To stabilise fixation an internal fixation stimulus is used. The image of the optic nerve head is positioned in the centre of the display. Image acquisition requires only 0.7 seconds. In the "customised mode," as a first step a polarimetric image is acquired from the macula area with the image of the fovea positioned in the centre of the screen. Data derived from the image of the macular area are then automatically subtracted from the peripapillary polarimetric image of the same eye, which is obtained as the second step of image acquisition. (In the GDx Access instrument used by us the macular images are not stored, and cannot be printed; and therefore cannot be used for further detailed analysis.) The resulting "corrected" image of the peripapillary retinal nerve fibre layer is then analysed automatically by the instrument's software in a similar way to the analysis used for images obtained with the conventional fixed angle corneal compensator.

In our investigation, polarimetric measurements with both the conventional (fixed angle compensator) image acquisition technique and the customised (individualised) corneal compensation technique were performed for each eye before surgery, and then 1 day and 6 days after the LASIK treatment. No patient was lost from follow up. Actual ametropia was corrected before each image acquisition using the software of the instrument. The optic nerve head contour 
line was placed exactly on the edge of the disc. The line of the 4 pixels-wide measuring ellipse was set at 1.75 disc diameters from the centre of the optic nerve head. Default quadrant positions were used. The definition of each polarimetric parameter calculated by the software and analysed by us is shown in Table 1. Both eyes were imaged, but only one randomly selected eye per participant was included in the evaluation.

ANOVA for repeated measurements was used to compare the RNFLT values recorded on the three different occasions. If the alteration evaluated with ANOVA was significant, the Scheffe test was used for paired comparison. The unpaired $t$ test was used for the comparison between the corresponding SLP-C and SLP-F values at baseline. Two way ANOVA was used to investigate the interaction between the thickness of the corneal flap and the alteration of the polarimetric values after LASIK. p Values of less than 0.05 were considered statistically significant.

\section{RESULTS}

The LASIK procedure and the postoperative period were uncomplicated in all eyes. It was possible to perform SLP with both techniques for each eye on all the three occasions. In the SLP-C mode the macular retardation images were always of regular bow tie pattern, with no obvious change in their appearance after LASIK.

The RNFLT values and the calculated polarimetric results as measured with the customised corneal polarisation compensation technique are shown in Table 2. The corresponding data obtained with the conventional technique using the fixed angle corneal compensator are presented in Table 3. The corresponding SLP-C and SLP-I values showed no difference at baseline (unpaired $t$ test, $\mathrm{p}>0.05$ ), except for ellipse standard deviation, which was significantly greater with SLP-F that with SLP-C $(p=0.026)$. After LASIK superior maximum thickness, inferior maximum thickness, normalised superior area, and normalised inferior area remained unchanged when measured with SLP-C (ANOVA, $p>0.05$ ). In contrast, superior maximum thickness and normalised superior area as measured with SLP-F decreased significantly after LASIK compared to the pre-LASIK values (Scheffe test, $\mathrm{p}<0.05$ ). Inferior maximum thickness measured with SLP-F tended to decrease after LASIK (Table 3).

Superior ratio and inferior ratio, maximal modulation, and ellipse modulation remained stable with SLP-F (Table 3,
ANOVA, $p>0.05$ ) but they all decreased after LASIK when measured with SLP-C (Table 2, Scheffe test, $\mathrm{p}<0.05$ ). Ellipse average thickness (available only in "customised mode") increased significantly after LASIK (Table 2). Superior to nasal ratio, symmetry of the superior, and inferior RNFLT as well as the number remained unchanged with either type of corneal compensation (ANOVA, $\mathrm{p}>0.05$ ). No value altered between the measurements made on the first day and the sixth day post-LASIK.

In the SLP-F mode a significant interaction was found between the thickness of the corneal flap and the alteration of several polarimetric parameters-namely the number, superior ratio, inferior ratio, superior/nasal ratio, maximum modulation, normalised superior area, normalised inferior area, and ellipse standard deviation (two way ANOVA, $p<0.05)$. In the SLP-C mode no interaction was found.

\section{DISCUSSION}

In the present study the potential benefit of individual corneal polarisation compensation for post-LASIK polarimetric RNFLT measurements was investigated by comparing the values obtained before surgery with those obtained 1 day and 6 days after uncomplicated LASIK on healthy ametropic eyes. The background of this investigation is that the RNFLT values, as measured with scanning laser polarimetry using the fixed angle corneal polarisation compensator (slow axis $15^{\circ}$ nasally downwards), have been consistently found to be reduced following uncomplicated LASIK. ${ }^{18-23}$

The clinical significance of the LASIK induced alterations of the polarimetric RNFLT is that this phenomenon may affect the clinical usefulness of the SLP technique in subjects who have undergone LASIK. Applanation tonometry measurements are irreversibly influenced by the thinning of the central cornea achieved by the LASIK procedure, and this results in false low intraocular pressure readings ${ }^{30}$ which are not suitable for glaucoma diagnosis or follow up. Therefore the SLP technique, if proved to be reliable after LASIK, will in future probably have a special practical importance in the diagnosis and follow up of glaucoma in patients who have been treated with LASIK.

The post-LASIK thinning of the polarimetric RNFLT measured with instruments using the fixed angle compensator seems to be caused by the corneal alterations induced by this refractive treatment. ${ }^{18-23}$ One may suppose that the transient stromal corneal oedema and the temporary

Table 1 Definition of the parameters analysed in this study

\begin{tabular}{|c|c|}
\hline Polarimetric parameter & Definition \\
\hline Superior maximum thickness & Average thickness $(\mu \mathrm{m})$ for the 210 thickest pixels in the superior quadrant \\
\hline Inferior maximum thickness & Average thickness $(\mu \mathrm{m})$ for the 210 thickest pixels in the inferior quadrant \\
\hline Normalised superior area & $\begin{array}{l}\text { The area under the superior hump of the RNFLT calculated after the subtraction of the average of the temporal and nasal } \\
\text { minimum values from the RNFLT values along the measuring ellipse }\end{array}$ \\
\hline Normalised inferior area & $\begin{array}{l}\text { The area under the inferior hump of the RNFLT calculated after the subtraction of the average of the temporal and nasal } \\
\text { minimum values from the RNFLT values along the measuring ellipse }\end{array}$ \\
\hline Superior thickness ratio & $\begin{array}{l}\text { The thickness ratio of the average of the } 200 \text { thickest pixels in the superior quadrant to the average for the } 200 \text { median pixels } \\
\text { in the temporal quadrant }\end{array}$ \\
\hline Inferior thickness ratio & $\begin{array}{l}\text { The thickness ratio of the average of the } 200 \text { thickest pixels in the inferior quadrant to the average for the } 200 \text { median pixels } \\
\text { in the temporal quadrant }\end{array}$ \\
\hline Superior/nasal ratio & $\begin{array}{l}\text { The thickness ratio of the average of the } 200 \text { thickest pixels in the superior quadrant to the average for the } 200 \text { median pixels } \\
\text { in the nasal quadrant }\end{array}$ \\
\hline Maximal modulation & The difference $(\mu \mathrm{m})$ between the thickest and the thinnest part of the retinal nerve fibre layer \\
\hline Ellipse modulation & The difference $(\mu \mathrm{m})$ between the thickest and the thinnest part of the retinal nerve fibre layer along the measuring ellipse \\
\hline Ellipse standard deviation & The standard deviation $(\mu \mathrm{m})$ of the RNFLT values along the measuring ellipse \\
\hline Ellipse average thickness* & Average thickness $(\mu \mathrm{m})$ for the pixels along the total measuring ellipse \\
\hline Symmetry & $\begin{array}{l}\text { The ratio of the average thickness of the thickest } 200 \text { pixels in the superior quadrant to the average for the thickest } 200 \text { pixels in } \\
\text { the inferior quadrant }\end{array}$ \\
\hline The number & An indicator of the probability that the eye suffers from glaucoma \\
\hline
\end{tabular}

*Available only in "customised mode." 
Table 2 Polarimetric values measured with customised corneal compensation at baseline and on days 1 and 6 after uncomplicated LASIK. The definitions of the parameters are shown in Table 1

\begin{tabular}{|c|c|c|c|c|c|c|c|}
\hline \multirow[b]{2}{*}{ SLP-C parameter } & \multicolumn{2}{|l|}{ Baseline (1) } & \multicolumn{2}{|l|}{ Day 1 (2) } & \multicolumn{2}{|l|}{ Day 6 (3) } & \multirow[b]{2}{*}{ p Value } \\
\hline & Mean (SD) & $95 \% \mathrm{Cl}$ & Mean (SD) & $95 \% \mathrm{Cl}$ & Mean (SD) & $95 \% \mathrm{Cl}$ & \\
\hline Superior maximum $(\mu \mathrm{m})$ & $78.1(9.6)$ & 72.84 to 83.45 & $80.2(8.4)$ & 75.48 to 84.85 & $79.1(9.3)$ & 74.76 to 85.07 & $p^{\#}=0.167$ \\
\hline Inferior maximum $(\mu \mathrm{m})$ & $82.9(12.4)$ & 76.04 to 89.80 & 83.7 (13.4) & 76.28 to 91.15 & $83.7(14.9)$ & 75.46 to 91.92 & $p^{\#}=0.918$ \\
\hline Normalised superior area & $0.123(0.029)$ & 0.107 to 0.139 & $0.127(0.023)$ & 0.114 to 0.139 & $0.125(0.021)$ & 0.114 to 0.137 & $p^{\#}=0.477$ \\
\hline Normalised inferior area & $0.141(0.024)$ & 0.128 to 0.154 & $0.145(0.028)$ & 0.129 to 0.160 & $0.143(0.030)$ & 0.126 to 0.159 & $\mathrm{p}^{\#}=0.585$ \\
\hline Superior ratio & $2.86(1.27)$ & 2.15 to 3.56 & $2.48(0.98)$ & 1.94 to 3.03 & $2.39(0.77)$ & 1.97 to 2.82 & $\begin{array}{l}\mathrm{p}^{*}{ }_{1-2}=0.108 \\
\mathrm{p}^{*}{ }_{1-3}=0.039 \\
\mathrm{p}^{*}{ }_{2-3}=0.881\end{array}$ \\
\hline Inferior ratio & $3.00(1.24)$ & 2.31 to 3.69 & $2.57(1.03)$ & 2.00 to 3.14 & $2.48(0.78)$ & 2.05 to 2.91 & $\begin{array}{l}p^{*}{ }_{1-2}=0.054 \\
p^{*}{ }_{1-3}=0.017 \\
p^{*}{ }_{2-3}=0.877\end{array}$ \\
\hline Superior/nasal ratio & $2.36(0.72)$ & 1.96 to 2.76 & $2.23(0.66)$ & 1.86 to 2.59 & $2.23(0.66)$ & 1.86 to 2.60 & $\mathrm{p}^{\#}=0.126$ \\
\hline Maximal modulation $(\mu \mathrm{m})$ & $2.40(1.06)$ & 1.82 to 2.99 & $1.97(0.88)$ & 1.48 to 2.45 & $1.89(0.61)$ & 1.55 to 2.23 & $\begin{array}{l}p^{*}{ }_{1-2}=0.063 \\
p^{*}{ }_{1-3}=0.024 \\
p^{*}{ }_{2-3}=0.906\end{array}$ \\
\hline Ellipse modulation $(\mu \mathrm{m})$ & $3.7(1.89)$ & 2.66 to 4.75 & $2.83(1.11)$ & 2.22 to 3.45 & $2.81(0.99)$ & 2.26 to 3.36 & $\begin{array}{l}\mathrm{p}^{*}{ }_{1-2}=0.035 \\
\mathrm{p}^{*}{ }_{1-3}=0.030 \\
\mathrm{p}^{*}{ }_{2-3}=0.997\end{array}$ \\
\hline Ellipse standard deviation & $21.52(3.85)$ & 19.39 to 23.66 & $20.80(3.50)$ & 18.86 to 22.74 & $20.52(3.07)$ & 18.82 to 22.22 & $\mathrm{p}^{\#}=0.301$ \\
\hline Ellipse average $(\mu \mathrm{m}) \dagger$ & $55.50(5.78)$ & 52.30 to 58.70 & $57.35(6.21)$ & 53.91 to 60.79 & $56.84(6.28)$ & 53.36 to 60.32 & $\begin{array}{l}p^{*}{ }_{1-2}=0.021 \\
p^{*}{ }_{1-3}=0.115 \\
p^{*}{ }_{2-3}=0.716\end{array}$ \\
\hline Symmetry & $0.95(0.10)$ & 0.90 to 1.00 & $0.97(0.12)$ & 0.91 to 1.03 & $0.97(0.12)$ & 0.91 to 1.03 & $\mathrm{p}^{\#}=0.476$ \\
\hline The number & $21.27(10.83)$ & 15.27 to 27.26 & $20.73(11.8)$ & 14.20 to 27.27 & $21.33(11.18)$ & 15.14 to 27.53 & $\mathrm{p}^{\#}=0.837$ \\
\hline
\end{tabular}

disturbance of the corneal surface, ${ }^{24} 25$ microfolds in the Bowman's layer and in the anterior stroma, ${ }^{26-29}$ and the occurrence of particles consisting of microscopic metallic debris from surgery, cellular debris, or inflammatory cells ${ }^{26-29}$ in the flap interface area are major factors in the early postoperative decrease of the polarimetric RNFLT values. These changes probably cause unstable alterations in the polarising property of the central cornea in the post-LASIK period, ${ }^{18}$ which cannot be consistently and completely neutralised with a fixed angle compensator system during the postoperative follow up. This hypothesis is supported by the interaction we found in the SLP-F mode between the alteration of several polarimetric parameters after LASIK and the thickness of the corneal flap. In the SLP-C mode there was no similar interaction, which suggests that the customised corneal polarisation compensation is superior than SLP-F for the neutralisation of the flap induced polarimetric artefacts.

In the present study pre-LASIK and early post-LASIK SLP measurements were performed on the same subjects with a scanning laser polarimeter supplied with both the conventional fixed angle corneal compensator and the customised corneal compensation system. This gave us the possibility to easily obtain both types of images and measurements at the same session, without even changing the patient's position. In order to show the potential differences between the results obtained with the two measurement techniques, the postLASIK measurements were performed on the first and the

Table 3 Polarimetric values measured with the fixed angle corneal compensator at baseline and on days 1 and 6 after uncomplicated LASIK

\begin{tabular}{|c|c|c|c|c|c|c|c|}
\hline \multirow[b]{2}{*}{ SLP-F parameter } & \multicolumn{2}{|l|}{ Baseline (1) } & \multicolumn{2}{|l|}{ Day 1 (2) } & \multicolumn{2}{|l|}{ Day 6 (3) } & \multirow[b]{2}{*}{ p Value } \\
\hline & Mean (SD) & $95 \% \mathrm{Cl}$ & Mean (SD) & $95 \% \mathrm{Cl}$ & Mean (SD) & $95 \% \mathrm{Cl}$ & \\
\hline Superior maximum $(\mu \mathrm{m})$ & $88.0(23.8)$ & 74.8 to 101.2 & $83.3(20.0)$ & 72.2 to 94.4 & $83.6(23.2)$ & 70.7 to 96.4 & $\begin{array}{l}p^{*}{ }_{1-2}=0.026 \\
p^{*}{ }_{1-3}=0.036\end{array}$ \\
\hline Inferior maximum ( $\mu \mathrm{m})$ & $93.0(29.0)$ & 76.9 to 109.1 & $90.1(24.4)$ & 76.6 to 103.6 & $90.1(24.9)$ & 76.3 to 103.8 & $\mathrm{p}^{\#}=0.089$ \\
\hline Normalised superior area & $0.113(0.037)$ & 0.093 to 0.134 & $0.104(0.040)$ & 0.082 to 0.126 & $0.105(0.042)$ & 0.082 to 0.128 & $\begin{array}{l}\mathrm{p}^{*}{ }_{1-2}=0.025 \\
\mathrm{p}^{*}{ }_{1-3}=0.061 \\
\mathrm{p}^{*}{ }_{2-3}=0.920\end{array}$ \\
\hline Normalised inferior area & $0.126(0.044)$ & 0.101 to 0.150 & $0.119(0.044)$ & 0.095 to 0.143 & $0.118(0.045)$ & 0.093 to 0.143 & $\begin{array}{l}\mathrm{p}^{\#}=0.039 \\
\mathrm{p}^{*}{ }_{1-2}=0.116 \\
\mathrm{p}^{*}{ }_{1-3}=0.061 \\
\mathrm{p}^{*}{ }_{2-3}=0.948\end{array}$ \\
\hline Superior ratio & $2.65(0.96)$ & 2.12 to 3.18 & $2.55(0.85)$ & 2.08 to 3.03 & $2.47(0.75)$ & 2.06 to 2.89 & $p^{\#}=0.403$ \\
\hline Inferior ratio & $2.76(0.97)$ & 2.23 to 3.30 & $2.76(0.94)$ & 2.23 to 3.28 & $2.66(0.77)$ & 2.24 to 3.09 & $\mathrm{p}^{\#}=0.722$ \\
\hline Superior/nasal ratio & $2.37(0.88)$ & 1.88 to 2.86 & $2.39(0.88)$ & 1.90 to 2.88 & $2.39(0.90)$ & 1.89 to 2.89 & $p^{\#}=0.957$ \\
\hline Maximal modulation $(\mu \mathrm{m})$ & $2.05(0.97)$ & 1.51 to 2.59 & $2.12(0.90)$ & 1.63 to 2.61 & $2.02(0.79)$ & 1.58 to 2.46 & $\mathrm{p}^{\#}=0.714$ \\
\hline Ellipse modulation $(\mu \mathrm{m})$ & $3.47(1.28)$ & 2.76 to 4.18 & $3.25(1.15)$ & 2.61 to 3.88 & $3.26(0.96)$ & 2.73 to 3.79 & $p^{\#}=0.615$ \\
\hline Ellipse standard deviation & $24.98(5.87)$ & 21.74 to 28.23 & $23.84(6.27)$ & 20.37 to 27.31 & $23.92(6.49)$ & 20.32 to 27.52 & $\mathrm{p}^{\#}=0.150$ \\
\hline Symmetry & $0.97(0.17)$ & 0.87 to 1.06 & $0.94(0.11)$ & 0.88 to 1.00 & $0.94(0.13)$ & 0.87 to 1.01 & $\mathrm{p}^{\#}=0.329$ \\
\hline The number & $19.13(16.77)$ & 9.85 to 28.42 & $17.00912 .46)$ & 10.10 to 23.90 & $18.40(15.52)$ & 9.81 to 26.99 & $\mathrm{p}^{\#}=0.675$ \\
\hline
\end{tabular}

$\mathrm{p}^{\#}=$ ANOVA for repeated measurements $\mathrm{p}^{*}$ Sheffe's test.

The definitions of the parameters are shown in Table 1. 
sixth postoperative days, since we found in our previous investigation $^{18}$ that SLP measurements in the first postoperative week were associated with the most pronounced RNFLT alterations.

With customised corneal polarisation compensation, the peripapillary polarimetric image is automatically corrected using data from the macular retardation image acquired as the first step of the measurement procedure. Since the fovea is free from retinal nerve fibres, the macular image represents the polarimetric effect only of the central cornea, the lens, and the retinal Henle layer. ${ }^{12}{ }^{14-16}$ The polarisation from the cornea is represented by a bow tie pattern distribution of the retardation around the fovea, ${ }^{12}{ }^{14-16}$ which shows a symmetric double hump distribution when the measuring ellipse is positioned around the fovea. ${ }^{12}{ }^{16}$ In our patients we were unable to detect any obvious change in the shape of the macular retardation images after LASIK compared to the preLASIK pattern, which suggests the polarimetric alterations due to LASIK were not caused by irregularities of the corneal polarisation pattern. However, since the instrument and the software we used provided no possibility for the detailed analysis of the macular images (these images were impossible to save or to print), we were not able to directly investigate the potential alteration of the axis or the magnitude of the macular retardation due to LASIK.

At baseline, before LASIK the corresponding values (except for ellipse standard deviation) did not differ in a statistically significant manner, though the mean values for the superior and inferior maximum thickness were higher for SLP-F than SLP-C. Ellipse standard deviation was significantly lower with the customised than with the fixed angle compensation. This shows that the elimination of the corneal polarisation did in fact influence the measured RNFLT. Using the SLP-F technique, similarly to our previous results ${ }^{18}$ and the results of other investigators who used instruments with the fixed corneal compensator, ${ }^{19-23}$ after LASIK the parameters representing the superior and inferior RNFLT decreased significantly or tended to decrease (Table 3). However, these parameters remained stable with customised corneal compensation (Table 2). These results suggest that customised corneal compensation prevents the LASIK induced virtual decrease of the measured RNLFT in the superior and inferior image quadrants, which represent the most vulnerable and the diagnostically most important sectors of the retinal nerve fibre layer in glaucoma.

In contrast with this stability of the superior and inferior sectors, after LASIK the superior to temporal ratio (superior ratio) and inferior to temporal ratio (inferior ratio) as well as maximal modulation and ellipse modulation, all decreased when measured with the customised compensation technique, but remained stable when measured with SLP-F (Tables 2 and 3). Since superior/nasal ratio showed no alteration with either measurement type (that is, retardation of the nasal quadrant remained stable), and the ellipse average thickness increased significantly after LASIK in SLP$\mathrm{C}$, it is logical to suppose that the retardation of the temporal quadrant as measured by the instrument, increased after LASIK. This unfortunately could not be verified by direct calculations, since the instrument provides no parameter to characterise the temporal RNFLT. The reason for the relative increase of retardation in the temporal sector combined with the preservation of retardation values in the other sectors remains unclear to us. One may speculate that the customised compensation, when used after LASIK, might not be optimal in this very reflective quadrant, in which the reproducibility of the polarimetric measurements has been found to be lower than in the other quadrants. ${ }^{131}$ The probable change of the retardation in the temporal quadrant represents a clear difference compared to the more uniform pattern of retardation decrease seen when using the SLP-F technique. Ratios using the retardation data of the temporal quadrant in the denominator are important in the diagnostic algorithm of the SLP instruments; these ratios decrease if the denominator value increases. The decrease of these ratios is similar to that seen in the progression of glaucoma but in that case it is caused by a decrease of the numerator value (that is, superior or inferior quadrant RNFLT). The observed postLASIK increase of the temporal sector retardation as measured with SLP-C is thus of clinical significance, and requires further investigation.

In summary, our findings show that customised corneal polarisation compensation avoids the LASIK induced virtual RNFLT thinning observed with polarimetric instruments working with the fixed angle corneal polarimetry compensator. However, the tendency for relative increase of the temporal quadrant retardation, as measured with the individual compensation method (but not with the fixed compensator) after uncomplicated LASIK surgery needs further investigation in order to prevent misinterpretation of the results obtained with the customised corneal compensator system.

\section{ACKNOWLEDGEMENTS}

This work was supported by Hungarian national grant for medical research no ETT 293/2000.

The authors have no financial interest in any instrument or technique used in the study. They thank Dr Csilla Palotás for technical assistance, and Peter Vargha for performing the statistical analysis.

\section{Authors' affiliations}

G Holló, A Katsanos, P Kóthy, I Süveges, 1st Department of

Ophthalmology, Semmelweis University, Budapest, Hungary

A Kerek, Optimum Private Laser Eye Institute, Budapest, Hungary

\section{REFERENCES}

1 Holló G, Süveges I, Nagymihály A, et al. Scanning laser polarimetry of the retinal nerve fibre layer in primary open angle and capsular glaucoma. Br J Ophthalmol 1997:81:857-61.

2 Weinreb RN, Zangwill L, Berry CC, et al. Detection of glaucoma with scanning laser polarimetry. Arch Ophthalmol 1998;116:1583-9.

3 Yamada N, Chen PP, Mills RP, et al. Glaucoma screening using the scanning laser polarimeter. J Glaucoma 2000;9:254-61.

4 Choplin NT, Lundy DC. The sensitivity and specificity of scanning laser polarimetry in the detection of glaucoma in a clinical setting. Ophthalmology 2001; 108:899-904.

5 Poinoosawmy D, Tan JCH, Bunce C, et al. The ability of the GDx Nerve Fibre Analyser neural network to diagnose glaucoma. Graefes Arch Clin Exp Ophthalmol 2001;239:122-7.

6 Poinoosawmy D, Tan JCH, Bunce C, et al. Longitudinal nerve fibre layer thickness change in normal-pressure glaucoma. Graefes Arch Clin Exp Ophthalmol 2000;238:965-9.

7 Holló G, Szabó A, Vargha P. Scanning laser polarimetry versus frequency doubling perimetry and conventional threshold perimetry: Changes during a 12 month follow-up in preperimetric glaucoma. Acta Ophthalmol Scand 2001;79:403-7.

8 Khong CH, Waldock A, Spry PGD, et al. Longitudinal measurements with scanning laser polarimetry and perimetry in ocular hypertension and glaucoma. Invest Ophthalmol Vis Sci 2001;42: S15, Abstract No 86.

9 Weinreb RN, Dreher AW, Coleman A. Histopathologic validation of Fourierellipsometry measurements of retinal nerve fiber thickness. Arch Ophthalmol 1990; 108:557-60.

10 Greenfield DS, Knighton RW, Huang X-R. Effect of corneal polarization axis on assessment of retinal nerve fiber layer thickness by scanning laser polarimetry. Am J Ophthalmol 2000;129:715-22.

11 Kogure S, Chiba T, Kinoshita T, et al. Effects of artefacts on scanning laser polarimetry of retinal nerve fibre layer thickness measurement. Br J Ophthalmol 2000:84:1013-7.

12 Zhou Q, Weinreb RN. Individualized compensation of anterior segment birefringence during scanning laser polarimetry. Invest Ophthalmol Vis Sci 2002;43:2221-8.

13 Weinreb RN, Bowd C, Greenfield DS, et al. Measurement of the magnitude and axis of corneal polarization with scanning laser polarimetry. Arch Ophthalmol 2002;120:901-6.

14 Knighton RW, Huang X-R, Greenfield DS. Analytical model of scanning laser polarimetry for retinal nerve fibre layer assessment. Invest Ophthalmol Vis Sci 2002:43:383-92. 
15 Greenfield DS, Knighton RW, Feuer WJ, et al Correction for corneal polarization axis improves the discriminating power of scanning laser polarimetry. Am J Ophthalmol 2002;134:27-33.

16 Garway-Heath D, Greaney MJ, Caprioli J. Correction for erroneous compensation of anterior segment birefringence with the scanning laser polarimeter for glaucoma diagnosis. Invest Ophthalmol Vis Sci 2002;43:1465-74.

17 Greenfield DS, Knighton RW. Stability of corneal polarization axis measurements for scanning laser polarimetry. Ophthalmology 2001;108:1065-9.

18 Holló G, Nagy ZZ, Vargha P, et al. Influence of post-LASIK corneal healing on scanning laser polarimetric measurement of the retinal nerve fibre layer thickness. Br J Ophthalmol 2002;86:627-31.

19 Gürses-Özden R, Pons ME, Barbieri C, et al. Scanning laser polarimetry measurements after laser-assisted in situ keratomileusis. Am $\mathbf{j}$ Ophthalmol 2000;129:461-4

20 Tsai $Y-Y$, Lin J-M. Effect of laser-assisted in situ keratomileusis on the retina nerve fiber layer. Retina 2000;20:342-5.

21 Gürses-Özden R, Liebmann JM, Schuffner D, et al. Retinal nerve fiber layer thickness remains unchanged following laser-assisted in situ keratomileusis. Am J Ophthalmol 2001;132:512-6.

22 Roberts TV, Lawless MA, Rogers CM, et al. The effect of laser-assisted in situ keratomileusis on retinal nerve fiber layer measurements obtained with scanning laser polarimetry. J Glaucoma 2002;11:173-7.
23 Kook MS, Lee S, Tchah $\mathrm{H}$ et al. Effect of laser assisted in situ keratomileusis on retinal nerve fiber layer thickness measurements by scanning laser polarimetry. J Cataract Refract Surg 2002;28:670-5

24 Battat L, Macri A, Dursun D, et al. Effects of laser in situ keratomileusis on tear production, clearance, and the ocular surface. Ophthalmology 2001; 108:1230-5.

25 Toda I, Asano-Kato N, Komai-Hori Y, et al. Dry eye after laser in situ keratomileusis. Am J Ophthalmol 2001;132:1-7.

26 Vesaluoma M, Pérez-Santonja J, Petroll WM et al. Corneal stromal changes induced by myopic LASIK. Invest Ophthalmol Vis Sci 2000:41:369-76.

27 Pisella P-J, Auzerie O, Bokobza Y, et al. Evaluation of corneal stroma changes in vivo after laser in situ keratomileusis with confocal microscopy. Ophthalmology 2001;108:1744-50.

28 Anderson NJ, Edelhauser HF, Sharara N, et al. Histologic and ultrastructural findings in human corneas after successful laser in situ keratomileusis. Arch Ophthalmol 2002; 120:288-93.

29 Mitooka K, Ramirez M, Maguire $\sqcup$, et al. Keratocyte density of central human cornea after laser in situ keratomileusis. Am J Ophthalmol 2002; 133:307-14.

30 Ginemo JAS, Muńoz LA, Valenzuela LA, et al. Influence of refraction on tonometric readings after photorefractive keratectomy and laser assisted in situ keratomileusis. Cornea 2000;19:512-6.

31 Holló G, Nagymihály A, Vargha P. Scanning laser polarimetry in corneal haze after excimer laser refractive surgery. J Glaucoma 1997;6:359-362.

\section{The lighter side}

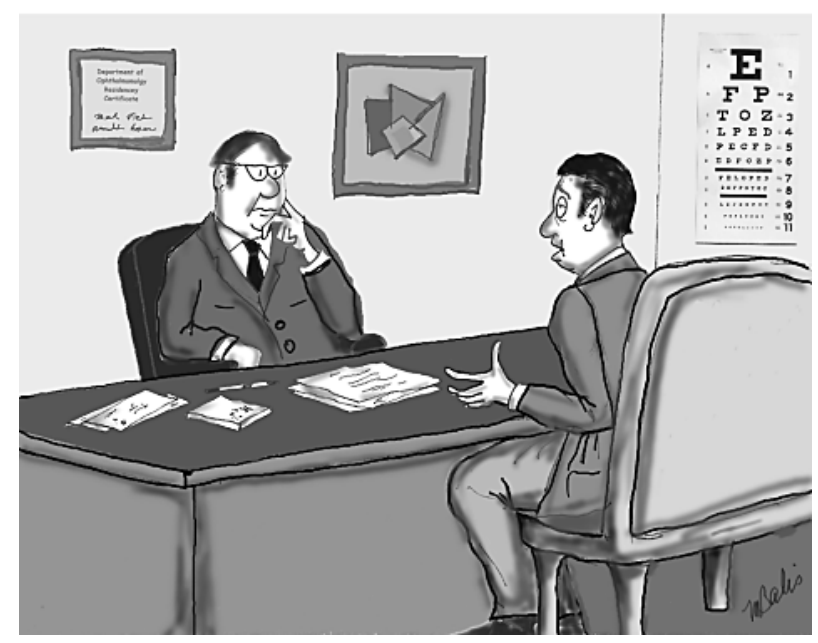

I want my cateracts back. I didn't realise how ugly my wife has become. (c) Michael Balis. 